\title{
Restoration of a Phosphaturic Response to Parathyroid Hormone in the Immature Rat
}

\author{
PAUL G. CORN, SUSAN E. MULRONEY, AND AVIAD HARAMATI
}

Department of Physiology and Biophysics, Georgetown University School of Medicine, Washington, DC 20007

\begin{abstract}
Recent studies have shown that immature rats display a diminished sensitivity to the phosphaturic effects of parathyroid hormone (PTH), and that the responsiveness to PTH increases with age. The attenuated phosphaturia may reflect an inability of the neonate to respond to the hormone because of functional immaturity of the developing kidney. Alternatively, PTH may actually inhibit tubular phosphate reabsorption in the neonate but, due to other phosphate conservation mechanisms, no phosphaturia occurs. Our objective was to determine whether a phosphaturic response to PTH would be elicited in immature rats during infusion of moderate amounts of phosphate (Pi). Clearance experiments were performed on 26 acutely thyroparathyroidectomized immature Wistar rats (3-5 wk of age) fed a normal $\mathrm{Pi}$ diet $(0.63 \%)$. In response to infusion of either Pi $(1 \mu \mathrm{mol} / \mathrm{min} \cdot 100 \mathrm{~g})$ (group I) or PTH $(8.3 \mathrm{ng} /$ min.100 g) (group II) alone, the fractional excretion of phosphate rose minimally (from $0.01 \pm 0.01 \%$ to $4.9 \pm$ $1.9 \%$ and from $0.12 \pm 0.12 \%$ to $2.9 \pm 1.4 \%$ for groups I and II, respectively). However, when $\mathrm{Pi}$ and $\mathrm{PTH}$ were combined either Pi first followed by PTH (group III) or PTH first followed by Pi (group IV), the fractional excretion of $P i$ rose dramatically (from $0.01 \pm 0.01$ to $21.8 \pm$ $3.5 \%$ and from $0.04 \pm 0.04$ to $27.7 \pm 3.3 \%$ for groups III and IV, respectively). A significant increase in urinary cAMP excretion occurred during infusion of PTH even when $\mathrm{Pi}$ excretion was minimal, but there was no further increase in urinary cAMP during the combined infusion of $\mathrm{Pi}$ and PTH. These results indicate that a phosphaturic response to PTH can be elicited in immature rats during infusion of moderate amounts of phosphate, and that the phosphaturia is dissociated from the increase in urinary excretion of cAMP. Thus, the attenuated phosphaturic response to PTH seen in developing rats is not due to functional immaturity of the kidney, but rather could be attributed to other factors that promote $P i$ reabsorption and prevent the phosphaturic effect from being expressed. (Pediatr Res 26: 54-57, 1989)
\end{abstract}

\section{Abbreviations}

PTH, parathyroid hormone

TPTX, thyroparathyroidectomized

$\mathrm{Pi}$, phosphate

The need for young, immature animals to maintain positive phosphate balance for growth is well recognized. This process

Received November 22, 1988: accepted March 17, 1989.

Correspondence and reprint requests to Dr. Aviad Haramati, Department of Physiology and Biophysics, Georgetown University School of Medicine, 3900 Reservoir Road, NW, Washington, DC 20007.

Supported by an NIH Research Career Development Award and Grant DK36111 to AH. P.G.C. was supported by BRSG Grant RR05360. may be facilitated by several adaptations in the developing kidney. Previous studies have shown that immature animals (1, 2 ) and newborn infants (3) have a relatively higher maximal rate of tubular phosphate reabsorption compared to adults. In addition, the neonate displays a relative hyporesponsiveness to stimuli which are normally phosphaturic in the adult, such as phosphate loading (2) and PTH $(1,4,5)$. Indeed, we recently reported that immature, acutely TPTX rats were less sensitive to the phosphaturic effects of PTH, and that the phosphaturic response increased progressively with age (6). Furthermore, this attenuated response was not associated with a diminished urinary excretion of cAMP, suggesting that the blunted response to the phosphaturic effects of PTH in the neonate is dissociated from the generation of CAMP. What remains unclear is whether the diminished sensitivity to the phosphaturic effects of PTH in the neonate represents a true adaptation as a consequence of the high metabolic demand for phosphate in a growing animal, or whether it is simply a reflection of a functionally immature nephron that is unable to respond to the hormone.

The purpose of these experiments was to further examine the mechanism by which developing animals resist the effects of PTH. Specifically, the objective was to determine whether a phosphaturic response could be elicited in immature rats during simultaneous infusion of moderate amounts of phosphate, i.e. which elevate the filtered load of phosphate without overwhelming the renal phosphate transport capacity. Such a manuever has been shown to restore a phosphaturic response to PTH in adult animals that were phosphate deprived (7), another model of renal resistance to $\mathrm{PTH}$. If in the presence of phosphate infusion a phosphaturic response to PTH is observed in immature rats, then this would suggest that the blunted response to PTH in these animals is not due to functional immaturity of the kidney, but rather to other factors serving to promote phosphate retention.

\section{MATERIALS AND METHODS}

Clearance experiments were performed on 26 male Wistar rats at $3-5$ wk of age $(94 \pm 3 \mathrm{~g})$. The animals were fed normal rat Chow (Ralston Purina, St. Louis, MO) containing 0.63\% Pi. All the animals were given food and water ad libitum and care was taken to ensure that the rats had not fasted.

On the day of the experiment, the rats were anesthetized with an intraperitoneal injection of Inactin $(100 \mathrm{mg} / \mathrm{kg}$ ) (Promonta, Hamburg, FRG) and prepared for renal clearance experiments. The animals were placed on a thermoregulated table and body temperature was monitored with a rectal probe and maintained at $37^{\circ} \mathrm{C}$ with a servocontrolled heat lamp. A tracheostomy was performed to maintain an unobstructed airway, and the animals were allowed to breathe spontaneously. Polyethylene catheters were placed in the carotid artery for blood pressure measurement and blood sampling, in the jugular vein for infusing solutions, and in the bladder for collecting urine samples.

All rats were TPTX by heat cautery to remove the influence 
of endogenous PTH. After TPTX, a 2-h recovery period elapsed to achieve steady state conditions, during which time a $2 \%$ inulin solution was infused at a rate of $2 \%$ body wt/h for the duration of the experiment. At this point one of four protocols was performed.

Group I: control, Pi, Pi. The purpose of this protocol was to evaluate the effects of $\mathrm{Pi}$ infusion alone, within the same time period as the group given both Pi and PTH (group III). After the recovery period, a 30-min control clearance (C) was taken. Subsequently, phosphate was added to the inulin infusion at a concentration calculated to deliver $1 \mu \mathrm{mol} / \mathrm{min} \cdot 100 \mathrm{~g}$ for the duration of the experiment. The phosphate solution was a $4: 1$ mixture of dibasic:monobasic sodium $\mathrm{Pi}$ salts adjusted to $\mathrm{pH}$ 7.4. A single bolus of $\mathrm{Pi}(30 \mu \mathrm{mol} / 100 \mathrm{~g})$ was administered at the onset of the $\mathrm{Pi}$ infusion. The infusion of phosphate proceeded for $20 \mathrm{~min}$ before sequential $30 \mathrm{~min}$ clearance periods were taken over the next $3 \mathrm{~h}$. Blood samples were obtained at the midpoint of each clearance period. The results represented as experimental period $1(\mathrm{Pi})$ are the mean of the clearances during the $1 \mathrm{st} h$ of $\mathrm{Pi}$ infusion, and the results represented as experimental period 2 (Pi) are the mean of the clearances obtained during the $3 \mathrm{rd} \mathrm{h}$ of Pi infusion.

Group II: control, PTH, PTH. The purpose of this protocol was to evaluate the effects of PTH infusion alone over the same time period as the group given both PTH and Pi (group IV). After the 2-h recovery period, a 30 -min control clearance (C) was obtained. Subsequently, PTH (synthetic, bovine 1-34 PTH, Peninsula Laboratories, Inc., Belmont, CA) was administered as a bolus injection $(249 \mathrm{ng} / 100 \mathrm{~g})$ and infused at $8.3 \mathrm{ng} / \mathrm{min} \cdot 100$ $\mathrm{g}$ for the duration of the experiment. The infusion of PTH continued for $40 \mathrm{~min}$ before consecutive 30 -min clearance periods were taken over the next $3 \mathrm{~h}$. The results given for experimental period 1 (PTH) are the mean of the clearances obtained during the 1 st $\mathrm{h}$ of PTH infusion, and the results given for experimental period 2 (PTH) are the mean of the clearances obtained during the $3 \mathrm{rd}$ h of PTH infusion.

Group III: control, Pi, Pi + PTH. The purpose of this protocol was to evaluate the renal effects of PTH given in the presence of ongoing $\mathrm{Pi}$ infusion. This protocol was similar to group I, except that PTH was administered concurrently with phosphate during the latter part of the experiment. Bolus and maintenance infusion doses for both Pi and PTH were calculated as described earlier for groups I and II. Data given for experimental period 1 ( $\mathrm{Pi}$ ) represents the mean of the clearances obtained during the infusion of phosphate alone, and the data given for experimental period $2(\mathrm{Pi}+\mathrm{PTH})$ represents the mean of the results obtained during the second hour of combined infusion.

Group IV: control, PTH, PTH + Pi. The purpose of this protocol was to evaluate the effects of $\mathrm{Pi}$ infusion given in the presence of ongoing PTH infusion. This protocol was similar to group II, except that Pi was administered with PTH during the latter part of the experiment. The data given for experimental period 1 (PTH) represents the mean of the clearances obtained during the infusion of PTH alone, and the data given for experimental period $2(\mathrm{PTH}+\mathrm{Pi})$ represents the mean of the results obtained during the 2 nd h of combined infusion.

Analysis. Inulin concentrations in plasma and urine samples were measured by the anthrone method (9). The glomerular filtration rate was equated with the clearance of inulin. Phosphate concentrations in plasma and urine samples were measured by the phosphomolybdate method described by Chen et al. (10), and CAMP concentrations in urine were determined by radioimmunoassay (New England Nuclear, Boston, MA).

All values are expressed as means $\pm S E$. Statistical comparisons were made with paired and unpaired Student's $t$-tests where appropriate.

\section{RESULTS}

Time control experiments: either Pi or PTH alone. The results obtained in the time control experiments from groups I and II are summarized in Table 1 . The infusion of $\mathrm{Pi}$ alone in immature TPTX rats led to a predictable increase in the plasma $\mathrm{Pi}$ concentration. However, the fractional excretion of $\mathrm{Pi}$ rose to a maximum of only $5 \%$ during the second period of $\mathrm{Pi}$ infusion. In all groups (I-IV), mean arterial blood pressures declined over the course of the experiment. However, the GFR remained stable.

The results from rats given only PTH (group II) are also presented in Table 1. Plasma Pi concentration did not change significantly with infusion of the hormone. The low fractional excretion of phosphate during the control period reflected the adequacy TPTX. During PTH infusion, fractional phosphate excretion rose to a maximum of $5.7 \%$, which is significantly attenuated to what has been previously obtained with this dose in older animals, i.e. fractional excretion of phosphate in the range of $20-30 \%(6)$.

Experiments in presence of both PTH and Pi. Table 2 contains the results obtained from group III rats, which received $\mathrm{Pi}$ infusion first followed by the combined administration of Pi and $\mathrm{PTH}$. Once again, the infusion of $\mathrm{Pi}$ alone led to a significant rise in the plasma $\mathrm{Pi}$ concentration, with only a minimal increase in the fractional excretion of Pi. After the addition of PTH, the fractional excretion of $\mathrm{Pi}$ increased markedly from 3.7 to $21.8 \%$. This occurred without a significant change in plasma Pi concentration.

In group IV, the reverse experiment was performed in which PTH was administered first, and then combined with Pi infusion. The results are given in Table 2 . As seen in the time control experiments of group II, there was no significant change in the plasma $\mathrm{Pi}$ concentration during the administration of PTH alone and the rise in the fractional excretion of $\mathrm{Pi}$ was again attenuated. However, with the simultaneous infusion of both PTH and Pi, there was a dramatic increase in fractional excretion of phosphate from 3.6 to $27.7 \%$.

To test whether the enhanced phosphaturic response to PTH was related to an augmentation in the signal transduction of the hormone at the level of the second messenger, determinations were made of the urinary excretion of cyclic AMP. The relationship between the renal excretion of cAMP and the fractional excretion of phosphate is shown in Figure 1. The values for urinary cAMP excretion during the control period or during infusion of phosphate alone were similar in all the groups. During infusion of PTH (group II and in experimental period 1 of group IV), there was a marked increase in urinary cAMP, but $\mathrm{Pi}$ excretion was minimal. During the combined infusion of PTH and $\mathrm{Pi}$ (groups III and IV), a significant increase in fractional $\mathrm{Pi}$ excretion was observed, but the urinary cAMP excretion rose to the same level as in group II. Accordingly, the lack of a phosphaturic response to PTH (when PTH was infused alone in groups II and IV) was not due to reduced generation of CAMP by PTH. By the same token, the onset of phosphaturia in the presence of both $\mathrm{PTH}$ and $\mathrm{Pi}$ was not due to an augmented cAMP response. Rather, it appears that the effects of PTH on Pi and cAMP excretion can be dissociated, and that the diminished phosphaturic response to $\mathrm{PTH}$ is not a consequence of an inability of the developing kidney to respond to the hormone.

\section{DISCUSSION}

The purpose of this study was to determine whether a phosphaturic response to PTH could be elicited in immature rats. The results of this study demonstrate that, whereas the infusion of $\mathrm{Pi}$ or $\mathrm{PTH}$ alone does not significantly alter Pi excretion in immature rats, the combination of $\mathrm{Pi}$ infusion and PTH, given in either order, results in a marked phosphaturia in these animals. Moreover, the combination of PTH and Pi infusion produced a phosphaturia that was much larger than the sum of the excretion of $\mathrm{Pi}$ induced by the administration of each stimuli alone. This points to a synergistic interaction between the effects of phosphate infusion and PTH in the immature kidney. 
Table 1. Effects of phosphate or PTH infusions alone on renal handling of phosphate in immature TPTX rats*

\begin{tabular}{cccccc}
\hline Period & $\begin{array}{c}\text { PPi } \\
(\mathrm{mM})\end{array}$ & $\begin{array}{c}\text { FEPi } \\
(\%)\end{array}$ & $\begin{array}{c}\text { FENa } \\
(\%)\end{array}$ & $\begin{array}{c}\text { GFR } \\
(\mathrm{ml} / \mathrm{min})\end{array}$ & $\begin{array}{c}\text { MAP } \\
(\mathrm{mmHg})\end{array}$ \\
\hline Group I & & & & & \\
C & $2.97 \pm 0.23$ & $0.01 \pm 0.01$ & $0.20 \pm 0.13$ & $0.09 \pm 0.09$ & $97 \pm 5$ \\
Pi & $3.96 \pm 0.25 \dagger$ & $0.18 \pm 0.06$ & $0.34 \pm 0.13$ & $1.09 \pm 0.09$ & $94 \pm 3$ \\
Pi & $4.69 \pm 0.28 \dagger$ & $4.93 \pm 1.90 \dagger$ & $0.15 \pm 0.04$ & $0.95 \pm 0.09$ & $83 \pm 3$ \\
Group II & & & & & $101 \pm 4$ \\
C & $3.17 \pm 0.15$ & $0.12 \pm 0.12$ & $0.04 \pm 0.02$ & $0.80 \pm 0.08$ & $98 \pm 3$ \\
PTH & $3.09 \pm 0.16$ & $5.71 \pm 1.75 \dagger$ & $0.13 \pm 0.02$ & $0.93 \pm 0.04$ & $92 \pm 3$ \\
PTH & $3.09 \pm 0.16$ & $2.93 \pm 1.36 \dagger$ & $0.21 \pm 0.11$ & $0.97 \pm 0.04$ & \\
\hline
\end{tabular}

* Values are means \pm SE from six animals in group I and six animals in group II. Pi, phosphate; PPi, plasma phosphate concentration; FEPi, fractional excretion of phosphate; FENa, fractional excretion of sodium; MAP, mean arterial pressure.

$\dagger p<0.05$ compared to the control period.

Table 2. Effects of combined infusion of phosphate and PTH on renal handling of phosphate in immature TPTX rats*

\begin{tabular}{cccccc}
\hline Period & $\begin{array}{c}\text { PPi } \\
(\mathrm{mM})\end{array}$ & $\begin{array}{c}\text { FEPi } \\
(\%)\end{array}$ & $\begin{array}{c}\text { FENa } \\
(\%)\end{array}$ & $\begin{array}{c}\text { GFR } \\
(\mathrm{ml} / \mathrm{min})\end{array}$ & $\begin{array}{c}\text { MAP } \\
(\mathrm{mmHg})\end{array}$ \\
\hline Group III & & & & & \\
C & $2.98 \pm 0.26$ & $0.01 \pm 0.01$ & $0.12 \pm 0.09$ & $1.25 \pm 0.18$ & $95 \pm 4$ \\
Pi & $4.17 \pm 0.35^{*}$ & $3.69 \pm 2.70^{*}$ & $0.52 \pm 0.20$ & $1.30 \pm 0.10$ & $97 \pm 2$ \\
Pi + PTH & $4.20 \pm 0.40^{*}$ & $21.75 \pm 3.46^{*}$ & $0.47 \pm 0.30$ & $1.27 \pm 0.08$ & $91 \pm 3$ \\
Group IV & & & & & \\
C & $2.95 \pm 0.24$ & $0.04 \pm 0.04$ & $0.19 \pm 0.08$ & $1.12 \pm 0.13$ & $97 \pm 4$ \\
PTH & $2.88 \pm 0.11$ & $3.60 \pm 1.20^{*}$ & $0.27 \pm 0.07$ & $1.14 \pm 0.10$ & $101 \pm 2$ \\
PTH + Pi & $3.30 \pm 0.20$ & $27.72 \pm 3.31^{*}$ & $0.25 \pm 0.11$ & $1.12 \pm 0.06$ & $95 \pm 5$ \\
\hline
\end{tabular}

* Values are means \pm SE from seven animals in group III and seven animals in group IV. Pi, phosphate; PPi, plasma phosphate concentration; FEPi, fractional excretion of phosphate; FENa, fractional excretion of sodium; MAP, mean arterial pressure.

$\dagger p<0.05$ compared to the control period.

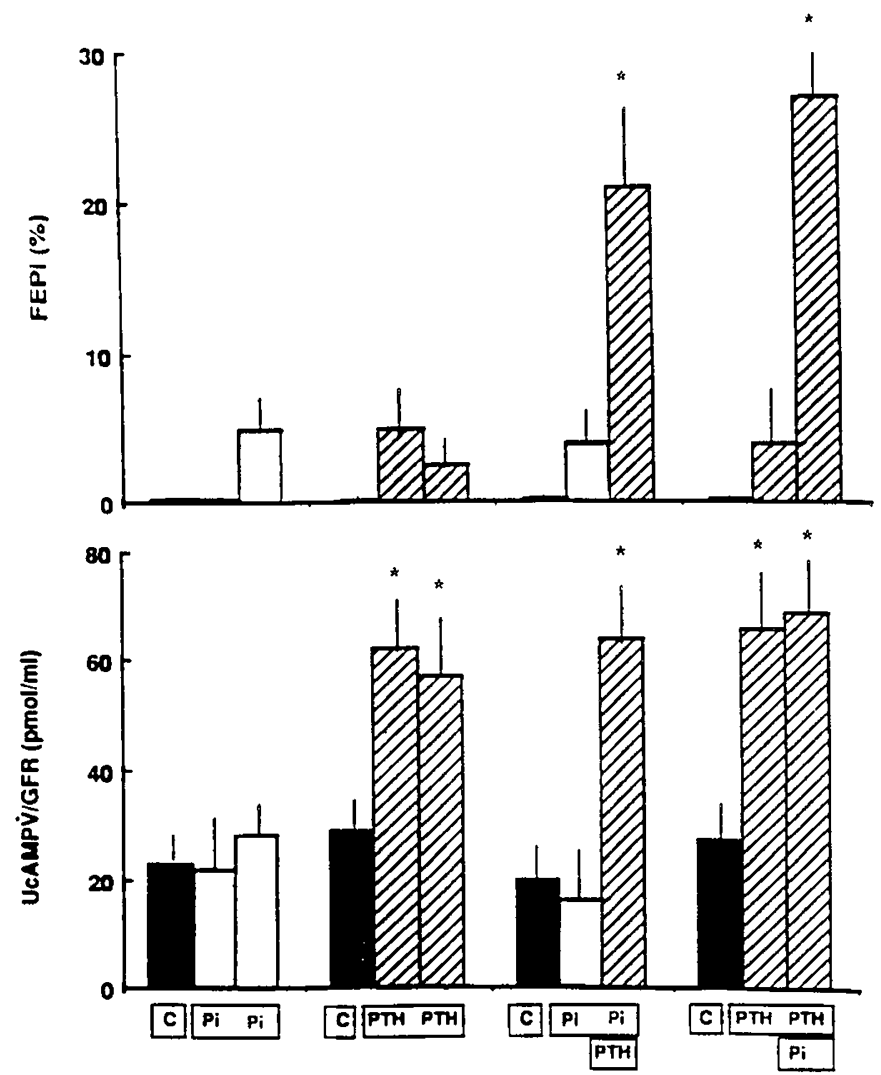

Fig. 1. Dissociation of the effects of PTH on the fractional excretion of phosphate (FEPi) and on the urinary excretion of cAMP (expressed as UcAMPV/ml of GFR) in immature, acutely TPTX rats. In every instance that PTH was administered (hatched bars), the urinary excretion of CAMP increased significantly (lower panel). However, an increase in FEPi (upper panel) occurred only during the combined infusion of phosphate $(\mathrm{Pi})$ and PTH.
The mechanism for the attenuated phosphaturic response to PTH in the immature kidney is still not clear. The notion that the blunted phosphaturic response in the newborn is related to a decrease in receptor number or sensitivity appears unlikely, in view of the significant increase in urinary cAMP excretion during infusion of PTH. Indeed, urinary cAMP excretion rose to a similar extent in each instance that PTH was administered, whether in the presence or absence of Pi infusion. The fact that a phosphaturia occurred only when PTH was combined with Pi infusion again demonstrates that the phosphaturic and urinary cAMP effects of PTH can be dissociated. Thus, these findings concur with previous suggestions that the renal PTH-adenylate cyclase-cAMP axis is intact in developing animals (11-13). However, the possibility that a defect might be present in the hormonal signal transduction at a step beyond cAMP generation cannot be excluded.

Spitzer et al. (14) have proposed that low intracellular $\mathrm{Pi}$ concentrations may be an important driving force for the avid reabsorption of $\mathrm{Pi}$ seen in immature animals. It is conceivable that under those conditions a favorable concentration gradient would exist across the luminal membrane to facilitate the transport of $\mathrm{Pi}$ into the tubular cell, perhaps even in the presence of PTH. Consequently, the infusion of Pi might limit this enhanced reabsorption by raising the intracellular Pi concentration. In our study, however, the magnitude of the phosphaturia in the presence of both PTH and Pi was similar even though, when Pi was added to an ongoing PTH infusion, the extent of repletion of intracellular Pi stores was, presumably, not as large as in the reverse experiment.

Perhaps the results can best be accounted for by an alternative explanation related to the nephron sites of $\mathrm{Pi}$ reabsorption. Although direct measurements of the segmental handling of $\mathrm{Pi}$ in immature rats are not available, data obtained in other states of $\mathrm{Pi}$ conservation may be applicable in this regard. Both $\mathrm{Pi}$ deprivation $(15,16)$ and respiratory alkalosis $(17)$ in adult rats are states characterized by an attenuated phosphaturic, but a normal CAMP, response to PTH. Results from several laboratories indicate that, although PTH still inhibits $\mathrm{Pi}$ reabsorption in 
the proximal convoluted tubule in these states, a phosphaturia does not occur because of enhanced $\mathrm{Pi}$ reabsorption in the pars recta and distal nephron segments (18-22). In a similar manner, moderate $\mathrm{Pi}$ infusions to $\mathrm{Pi}$-deprived adult rats, at rates which do not raise $\mathrm{Pi}$ excretion, also have been shown to decrease $\mathrm{Pi}$ uptake in proximal tubule brush border membrane vesicles (23), and increase the delivery of phosphate out of the proximal convoluted tubule (24). Because of the many similarities in the renal handling of phosphate between young, rapidly growing rats and Pi-deprived adult rats, it is reasonable to propose that nephron sites beyond the proximal tubule reabsorb significant amounts of $\mathrm{Pi}$ in the developing kidney. Accordingly, when PTH or Pi were administered separately, some inhibition of $\mathrm{Pi}$ reabsorption in the proximal tubule may have occurred. However, a phosphaturia did not ensue because the more distal sites could reabsorb the increased load of Pi. In contrast, when $\mathrm{Pi}$ and PTH were combined, the magnitude of the load delivered to the pars recta and distal tubule could have overwhelmed the Pi transport capacity of those segments, resulting in the increase in Pi excretion that was observed. This notion would also explain why a phosphaturia of similar magnitude occurred even when the order of administration of Pi and PTH was reversed.

Bonjour and Fleisch (25) have proposed that the tubular reabsorption of $\mathrm{Pi}$ is regulated by the balance between the demand of the animal for phosphate and the available dietary Pi supply. This thesis is clearly reflected in the parallels between the renal handling of $\mathrm{Pi}$ in immature rats fed normal $\mathrm{Pi}$ diet and $\mathrm{Pi}$ deprived adult rats. Both the immature rats (that have an increased demand for Pi due to an accelerated rate of growth) and $\mathrm{Pi}$-deprived adult rats (that have a reduced supply of phosphate) exhibit an elevated tubular capacity to reabsorb $\mathrm{Pi}(2,15)$ and an apparent reduced sensitivity to $\mathrm{PTH}(6,16)$; adaptations that serve to facilitate the renal conservation of $\mathrm{Pi}$.

In summary, the results of this study indicate that a phosphaturic response to PTH can be elicited in immature rats by combined infusion of $\mathrm{Pi}$ and $\mathrm{PTH}$, and that the phosphaturia is dissociated from the increase in urinary excretion of cAMP. The findings also suggest that the attenuated phosphaturic response to PTH seen in the neonate does not represent functional immaturity of the developing kidney, but rather may serve as an appropriate adaptation that contributes to $\mathrm{Pi}$ retention during growth and development.

\section{REFERENCES}

1. Johnson V, Spitzer A 1986 Renal reabsorption of phosphate during development: whole kidney events. Am J Physiol 251:F251-F256

2. Haramati A, Mulroney SE, Webster SK 1988 Developmental changes in the tubular capacity for phosphate reabsorption in the rat. Am J Physiol 255:F287-F291
3. Brodehl J, Gellisen K, Weber H-P 1982 Postnatal development of tubular phosphate reabsorption. Clin Nephrol 17:163-171

4. Linarelli LG 1972 Newborn urinary cyclic AMP and developmental renal responsiveness to parathyroid hormone. Pediatrics 50:14-23

5. Erdem G, Tuncer M, Saatci U, Ciliv G 1982 Evaluation of the renal responsiveness to exogenous parathyroid hormone on the first and third days of life. Turk J Pediatr 24:13-19

6. Webster SK, Haramati A 1985 Developmental changes in the phosphaturic response to parathyroid hormone in the rat. Am J Physiol 249:F251-F255

7. Wong NLM, Quamme GA, O'Callaghan TJ, Sutton RAL, Dirks JH 1980 Renal tubular transport in phosphate depletion: A micropuncture study. Can J Physiol Pharmacol 58:1063-1071

8. Haramati A, Kuechle J, Knox FG 1983 Restoration of phosphaturia in phosphate-deprived rats in the presence of both parathyroid hormone and phosphate infusion. Proc Soc Exp Biol Med 172:163-167

9. Fuhr J, Kaczmarzyk J, Kruttgen CD 1955 Eine einefache colorimetrische Methode zur Inulinbestimmung fur Nierenclearanceuntersuchungen bei Stoffwechselgesunden und Diabetikern. Klin Wochenschr 33:729-730

10. Chen PS, Toribara TY, Warner H 1956 Microdetermination of phosphorus. Anal Chem 28:1756-1758

11. Linarelli LG, Bobik J, Bobik C 1973 The effect of parathyroid hormone on rabbit renal cortex adenyl cyclase during development. Pediatr Res 7:878882

12. Muschek LD, Hook JB 1973 Changes in adenosine 3',5'-monophosphate induced by parathyroid hormone and theophylline in the developing rat renal cortex. Dev Biol 33:56-61

13. Thomas ML, Anast CS, Forte LR 1981 Regulation of calcium homeostasis in the fetal and neonatal rat. Am J Physiol 240:E367-E372

14. Spitzer A, Kaskel FJ, Feld LG, Trachtman H, Johnson V, Choi Y, Kumar AM 1983 Renal regulation of phosphate homeostasis during growth. Semin Nephrol 3:87-93

15. Troehler U, Bonjour JP, Fleisch H 1976 Inorganic phosphate homeostasis. Renal adaptation to the dietary intake in intact and thyroparathyroidectomized rats. $\mathrm{J}$ Clin Invest 57:264-273

16. Steele TH 1976 Renal resistance to parathyroid hormone during phosphate deprivation. J Clin Invest 58:1461-1464

17. Hoppe A, Metler M, Berndt TJ, Knox FG, Angielski S 1982 Effect of respiratory alkalosis on renal phosphate excretion. Am J Physiol 243:F47 I-F475

18. Pastoriza-Munoz E, Mishler DR, Lechene C 1983 Effect of phosphate deprivation on phosphate reabsorption in rat nephron:role of PTH. Am J Physiol 244:F140-F149

19. Haas JA, Berndt TJ, Haramati A, Knox FG 1984 Nephron sites of action of nicotinamide on phosphate reabsorption. Am J Physiol 246:F27-F31

20. Webster SK, Haramati A, Knox FG 1986 Effect of dexamethasone on segmental phosphate reabsorption in phosphate-deprived rats. Am $J$ Physiol 251:F576-F580

21. Berndt TJ, Knox FG 1985 Nephron site of resistance to the phosphaturic effect of PTH during respiratory alkalosis. Am J Physiol 249:F919-F922

22. Berndt TJ, Onsgard MJ, Knox FG 1988 Effect of cAMP analogue infusion on phosphate reabsorption in phosphate-deprived rats. Am J Physiol 255:F96F99

23. Cheng L, Dersch C, Kraus E, Spector D, Sacktor B 1984 Renal adaptation to phosphate load in the acutely thyroparathyroidectomized rat: Rapid alteration in brush border membrane phosphate transport. Am $\mathbf{J}$ Physio 246:F488-F494

24. Awazu M, Berndt TJ, Knox FG 1987 Effect of phosphate infusion on proximal tubule phosphate reabsorption in phosphate-deprived and respiratory alkalotic rats. Mineral Electrolyte Metab 13:393-396

25. Bonjour JP, Fleisch H 1980 Tubular adaptation to the supply and requirements of phosphate. In: Massry SG, Fleisch H (eds) Renal Handling of Phosphate. Plenum, New York, pp 243-264 